\title{
CLAUDICAÇÃO NA CRIANÇA
}

\author{
LIMPING IN CHILDREN
}

\begin{abstract}
Cláudio Santili ${ }^{1}$, Wilson Lino Júnior ${ }^{2}$, Ellen de Oliveira Goiano ${ }^{3}$, Romero Antunes Barreto Lins ${ }^{4}$, Gilberto Waisberg ${ }^{5}$,
\end{abstract} Susana dos Reis Braga', Miguel Akkari ${ }^{7}$

\section{RESUMO}

A claudicação na criança é uma queixa frequente nos consultórios dos pediatras, dos ortopedistas pediátricos e nos pronto-socorros. Diversas são as causas para essa condição e identificá-las torna-se um desafio. Quanto maior a idade do paciente, melhor é a coleta da anamnese e mais completo se torna o exame físico, facilitando o raciocínio médico na busca da origem do distúrbio. Para facilitar a abordagem, três grupos etários podem e devem ser considerados. No grupo etário infantil, de um aos três anos de idade, os diagnósticos mais prováveis incluem: sinovite transitória, artrite séptica, desordens neurológicas (paralisia cerebral (PC) branda e distrofia muscular), displasia do desenvolvimento do quadril (DDQ), coxa vara, artrite reumatoide juvenil (ARJ) e neoplasias (osteoma osteoide, leucemia); no grupo etário escolar, dos quatro aos 10 anos de idade, além dos diagnósticos acima, doença de Legg-Calvé-Perthes, menisco discoide, discrepâncias de comprimento dos membros inferiores e "dor do crescimento"; nos adolescentes, dos 11 aos 15 anos de idade: epifisiólise, DDQ, condrólise, síndromes de overuse, osteocondrite dissecante e coalizão tarsal. A proposta deste trabalho é atualizar o leitor em relação à abordagem do paciente pediátrico que apresenta claudicação e discutir as suas prováveis causas.

Descritores - Marcha; Criança; Claudicação intermitente; Doença de Legg-Perthes; Quadril; Osteocondrite dissecante; Artrite reumatoide juvenil; Artrite infecciosa; Paralisia cerebral; Sinovite

\section{ABSTRACT}

Limping in children is a common complaint at pediatric, pediatric orthopaedic offices and in emergency rooms. There are several causes for this condition, and identifying them is a challenge. The older the patient, the better the anamnesis and more detailed the physical examination will be, enabling an easier medical assessment for searching the source of the disorder. In order to make the approach easier, three age groups can and should be considered. Among infants (1 to 3 years old), diagnosis will most likely be: transitory synovitis, septic arthritis, neurological disorders (mild brain palsy (BP) and muscular dystrophy), congenital hip dislocation (CHD), varus thigh, juvenile rheumatoid arthritis (JRA) and neoplasias (osteoid osteoma, leukemia); in the scholar age group, between 4 and 10 years old, in addition to the diagnoses above, Legg-Calvé-Perthes disease, discoid meniscus, inferior limbs discrepancy and unspecific muscular pain; in adolescents (11 to 15 years old): slipped capital femoral epiphysis, congenital hip dislocation, chondrolysis, overuse syndromes, dissecans osteochondritis, and tarsal coalition. The purpose of this study is to provide an update on how to approach pediatric patients presenting with limping, and to discuss its potential causes.

Keywords - Gait; Child; Intermittent claudication; Legg-Perthes disease; Hip; Osteochondritis dissecans; Arthritis, juvenile rheumatoid; Arthritis, Infectious; Cerebral palsy; Synovitis

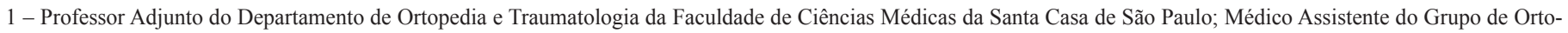
pedia e Traumatologia Pediátrica da Irmandade da Santa Casa de Misericórdia de São Paulo, São Paulo, Brasil.

2 - Médico Assistente do Grupo de Ortopedia e Traumatologia Pediátrica da Irmandade da Santa Casa de Misericórdia de São Paulo, São Paulo, Brasil.

3 - Médica Estagiária do Grupo de Ortopedia e Traumatologia Pediátrica da Irmandade da Santa Casa de Misericórdia de São Paulo, São Paulo, Brasil.

4 - Médico Residente (R3) do Departamento de Ortopedia e Traumatologia da Faculdade de Ciências Médicas da Santa Casa de São Paulo, São Paulo, Brasil.

5 - Médico Assistente do Grupo de Ortopedia e Traumatologia Pediátrica da Irmandade da Santa Casa de Misericórdia de São Paulo, São Paulo, Brasil.

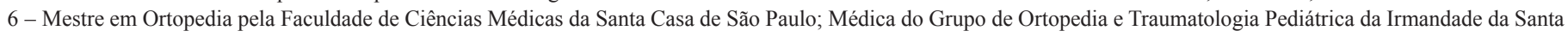
Casa de Misericórdia de São Paulo, São Paulo, Brasil.

7 - Chefe do Grupo de Ortopedia e Traumatologia Pediátrica da Irmandade da Santa Casa de Misericórdia de São Paulo, São Paulo, Brasil.

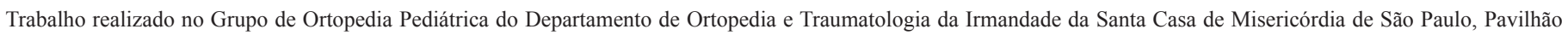
"Fernandinho Simonsen" (Diretor: Prof. Osmar Avanzi).

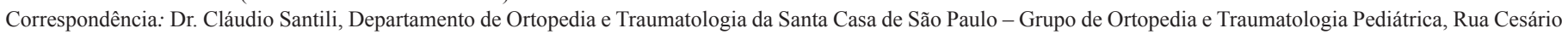
Mota Junior, 112 - 01277-900 - São Paulo, SP. E-mail: ortopediatrica@yahoo.com.br 


\section{INTRODUÇÃO}

O diagnóstico das condições que acarretam claudicação na criança é um desafio ${ }^{(1,2)}$, mesmo para profissionais mais experientes e que estejam acostumados a lidar com pacientes que não conseguem dar maiores informações. Normalmente, os distúrbios que causam alteração da marcha podem ser divididos de acordo com o padrão da claudicação e a idade em que aparecem ${ }^{(3)}$. Para facilitar a abordagem do pediatra, que normalmente é o primeiro médico a ser procurado, três grupos etários devem ser considerados: infantil, que inclui as crianças que estão aprendendo a caminhar (um a três anos); escolar, com padrão de marcha mais maduro (quatro a 10 anos); e adolescentes (11 a 15 anos) ${ }^{(4)}$.

Assim, deve ser realizada uma abordagem sistematizada para cada faixa etária, de forma a se obter uma avaliação mais precisa e orientada para as afecções próprias da idade, inclusive para se ter em mente quais os exames a serem solicitados em cada caso, otimizando a probabilidade de se obter o diagnóstico precoce ${ }^{(5)}$.

Em relação à criança, é preciso observar que elas iniciam a deambulação com apoio em torno dos 12 meses e aos 18 meses, em geral, se tornam independentes para a marcha, porém, com movimentos ainda descoordenados, mantendo um padrão imaturo até por volta dos cinco anos de idade e chegam ao padrão adulto aos sete anos.

Muitas vezes, a claudicação está relacionada com a dor e o padrão antálgico da marcha observado é caracterizado por passos rápidos e descarga mínima do peso na extremidade dolorosa, encurtando a fase de apoio. Esse é o tipo mais comum de claudicação ${ }^{(6,7)} \mathrm{e}$, em geral, é facilmente constatado através do exame físico dinâmico e complementado com a inspeção de sinais de localização como os flogísticos e os pós-traumáticos, muitas vezes presentes, e pela palpação cuidadosa das áreas envolvidas em que a criança acusa a dor. O tipo antálgico, secundário ao trauma, não será abordado neste artigo.

\section{PERÍODO INFANTIL - DE UM AOS TRÊS ANOS DE IDADE}

Esses pacientes são os que acarretam maior dificuldade diagnóstica no que diz respeito aos problemas da marcha $^{(2,8,9)}$. Trata-se de um grupo que, pela pouca idade, é pouco colaborador, tanto em relação às informações colhidas na anamnese, que na maioria das vezes é obtida somente através das queixas dos pais, quanto na realização do exame físico.
Outro dado importante e de que não devemos esquecer é que nessa idade as crianças apresentam um padrão de marcha imaturo ${ }^{(10,11)}$, caracterizado pela base alargada, aumento da flexão dos quadris e joelhos e braços ao lado do corpo com o cotovelo estendido, tudo isso para melhorar a fase de balanço, naturalmente desequilibrada. Como não conseguem aumentar o tamanho do passo, em virtude da falta de maturidade neuromuscular, aumentam sua cadência, objetivando ganhar velocidade. Toda essa variação no padrão do caminhar deve ser avaliada e considerada, na hora de determinar se verdadeiramente existe claudicação.

Se, de fato, for verificada alteração, os diagnósticos mais prováveis relacionados com esse grupo de crianças incluem: sinovite transitória, artrite séptica, desordens neurológicas (paralisia cerebral (PC) branda e distrofia muscular), displasia do desenvolvimento do quadril (DDQ), coxa vara, artrite reumatoide juvenil (ARJ) e neoplasias (osteoma osteoide, leucemia) ${ }^{(7,11,12)}$.

\section{Distúrbios inflamatórios/infecciosos}

Sinovite transitória $X$ artrite séptica - Essas duas condições levam a um quadro agudo de claudicação dolorosa que causa dúvida em seu diagnóstico diferencial $^{(13,14)}$; devem ser bem distinguidas uma da outra pela sua evolução que, em geral, é favorável no caso da sinovite e desastrosa no caso da pioartrite, se não forem tomadas as devidas providências. O local preferencial de acometimento é o quadril. A sinovite transitória apresenta um quadro de claudicação que varia de leve a moderado, mas a criança normalmente não deixa de fazer suas atividades habituais, porém, o quadro doloroso pode se arrastar por dias (cerca de sete a dez dias) até a resolução, que ocorre gradual e espontaneamente na maioria dos casos. Crianças com artrite séptica tendem a ser muito mais irritadas e menos colaboradoras, podendo inclusive se negar a caminhar, além de geralmente apresentar comprometimento do estado geral. A mobilização da articulação durante o exame físico está normalmente mais limitada na artrite séptica, mas, se ainda assim houver dúvida, exames laboratoriais devem ser solicitados.

A contagem de leucócitos ao hemograma, a velocidade de hemossedimentação (VHS) e a proteína $C$ reativa (PCR), geralmente estão dentro da faixa de normalidade na sinovite, enquanto que na artrite séptica estão elevadas. Febre alta $\left(>38,5^{\circ} \mathrm{C}\right)$ também é considerada um fator preditivo positivo importante de artrite séptica $^{(14)}$. 
Se estiverem discretamente elevados ou normais, porém limítrofes, e o quadro clínico for mais intenso, a punção articular se faz necessária para a elucidação ${ }^{(6,15)}$. A contagem de glóbulos brancos na análise do material puncionado revela-se entre 80.000 e 200.000 com mais de $75 \%$ de polimorfonucleares na artrite séptica, enquanto na sinovite transitória fica em torno de 5.000 a 15.000 , com menos de $25 \%$ de polimorfos. Coloração de Gram também deve ser solicitada na análise do material para ajudar na seleção do antibacteriano a ser utilizado no caso de a artrite ser confirmada, mesmo sabendo-se que o $S$. aureus é o patógeno mais comum; até o isolamento em cultura, outros agentes como Streptococcus do grupo B, Pseudomonas aeruginosa e Haemophilus influenza devem ser considerados.

Artrite reumatoide juvenil (ARJ) - A criança que no início da marcha apresenta claudicação dolorosa leve e insidiosa, em torno dos dois anos de idade, pode estar manifestando os primeiros sintomas da forma pauciarticular de ARJ. Essa forma, que também é a mais comum, tem incidência maior em meninas, na proporção de $4: 1$; as articulações mais frequentemente acometidas são os joelhos e os tornozelos; é acompanhada de edema, calor local e limitação da amplitude de movimentos articulares.

Os exames laboratoriais como leucograma, VHS, fator reumatoide e o ANA (anticorpo antinuclear) podem estar normais durante o quadro inicial em até $50 \%$ dos casos, o que não deve descartar o diagnóstico ${ }^{(16)}$.

O quadro clínico em geral é intermitente, melhorando com repouso, analgésicos e restrição da atividade, porém, se o edema persistir, um reumatologista pediátrico deverá ser consultado.

\section{Distúrbios neurológicos}

Paralisia cerebral (PC) - A maioria das crianças em torno dos 12 meses de vida começa a dar seus primeiros passos e evolui para marcha sem auxílio, dentro da faixa de normalidade, por volta dos 18 meses. Se a deambulação atrasar para além dessa idade ou se ela estiver desde o início anormal, o mais provável é que uma desordem neurológica esteja presente.

A disfunção neurológica mais comum, que pode passar despercebida antes dos primeiros passos da criança e que leva à claudicação durante a deambulação, é a paralisia cerebral branda ${ }^{(6)}$.

Nesses pacientes o desbalanço muscular é menor, o que pode gerar dúvidas no diagnóstico, diferentemente do que ocorre na marcha espástica característica da
PC. Porém, um bom exame clínico mostrará limitação da amplitude de movimento do joelho e do tornozelo, hiperreflexia e presença de clônus, que diferenciarão o problema. A partir daí, os pais devem ser orientados sobre a condição e a criança encaminhada para um centro multiespecializado de tratamento, com o ortopedista pediátrico conduzindo os princípios terapêuticos.

Distrofia muscular - Nessa condição incomum, o atraso no início da marcha é acompanhado de um histórico de tropeços de repetição, quedas frequentes e dificuldade para subir escadas devido à fraqueza da musculatura proximal da raiz do membro (principalmente glúteo máximo, glúteo médio e quadríceps), a panturrilha, em consequência, aparenta falsa hipertrofia e o clássico sinal de Gowers, em que a criança colocada de bruços é solicitada a se levantar e o faz "escalando sobre si mesma" (Figura 1).
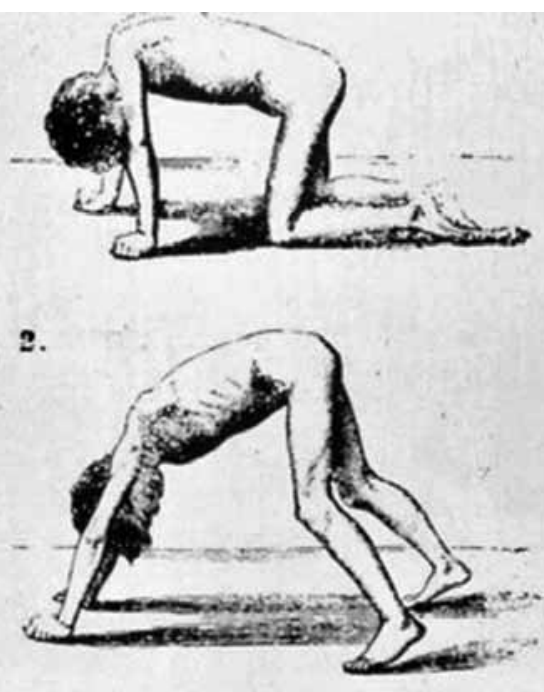

3.

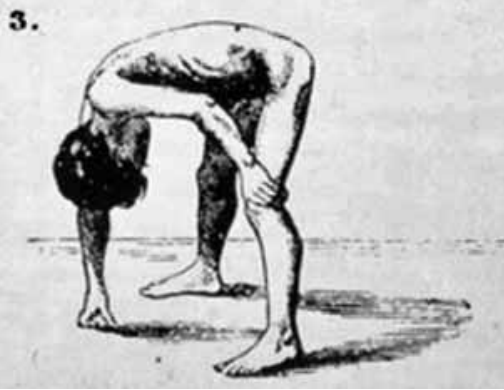

Figura 1 - Sinal de Gowers (Extraído de Gowers WR. A manual of disease of the nervous system. London: Churchill, 1886;1:391-4).

Como prova diagnóstica, a dosagem de creatina fosfoquinase sérica (CPK) pode ser solicitada nos primeiros estágios da distrofia, podendo estar alterada de 200 a 300 vezes o normal ${ }^{(17)}$. 
Em geral, o paciente é levado ao consultório por volta dos três aos seis anos; acomete quase que exclusivamente meninos, já que é uma herança recessiva ligada ao cromossomo $X$.

Pode existir história familiar positiva, a doença é progressiva e evolui lentamente. Os pacientes geralmente morrem durante a segunda ou terceira década de vida por insuficiência respiratória ou cardíaca.

\section{Distúrbios do desenvolvimento/congênitos}

Displasia do desenvolvimento do quadril (DDQ) Quando passa despercebida ao nascimento e durante os primeiros meses de vida, persistindo além da idade da marcha sem diagnóstico, essa condição atrasa o inicio da deambulação e causa um tipo de claudicação indolor. Pode ser uni ou bilateral; quando unilateral, o membro afetado encontra-se encurtado e a criança anda na ponta do pé, a abdução do quadril é limitada, com tensão dos adutores e pode haver leve contratura em flexão do quadril. Quando bilateral, para deambular a criança aumenta a lordose lombar e a marcha se assemelha à "marcha de pato" (marcha anserina) e o balanço do tronco pela limitação da abdução e insuficiência do glúteo médio bilateralmente (sinal de Trendelenburg positivo). A radiografia em AP da bacia confirma facilmente o diagnóstico nessa idade, evidenciando a luxação ou subluxação dos quadris, não sendo necessários outros exames de imagem (Figuras 2 e 3).

Coxa vara - O quadro clínico aqui, independente de a condição ser congênita ou do desenvolvimento, assemelha-se ao da DDQ, porém, é muito menos comum, na proporção de 1:20. Ao exame clínico, o que a diferencia da luxação congênita do quadril é a proeminência na região do trocanter maior, secundária à fraqueza funcional do músculo, e a rotação interna, que também

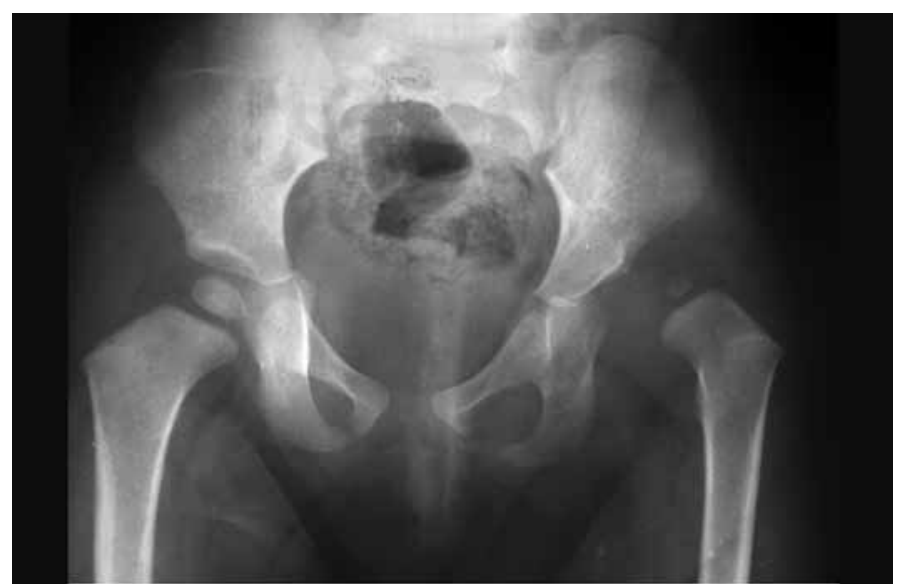

Figura 2 - Radiografia em AP com luxação do quadril à E

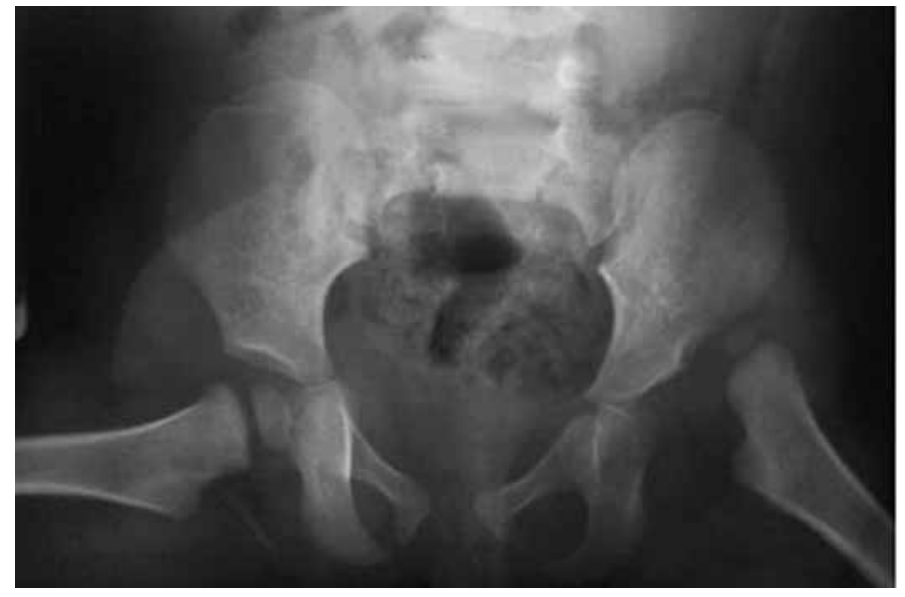

Figura 3 - Radiografia na "posição de rã"

está limitada nas fases mais avançadas; entretanto, o diagnóstico conclusivo é feito mediante a radiografia da bacia em AP (Figura 4), onde não se observa a luxação, porém, o colo se encontra em posição quase perpendicular em relação à diáfise do fêmur e a placa de crescimento está verticalizada.

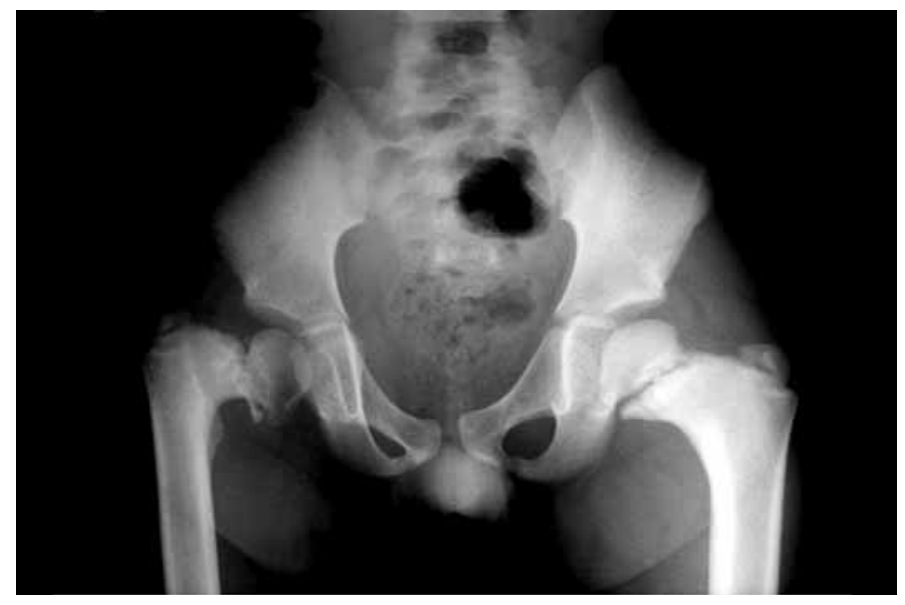

Figura 4 - Coxa vara do desenvolvimento

\section{Distúrbios neoplásicos}

Osteoma osteoide - É incomum em crianças antes dos cinco anos de idade, porém, quando presente, seu diagnóstico é um desafio, especialmente no início da idade da marcha. Provoca claudicação dolorosa, predominantemente noturna e pode passar despercebido nas radiografias. $\mathrm{O}$ exame clínico é inocente, dor à palpação não é usual, porém, como regra geral, há melhora importante com o uso de salicilatos (AAS), o que pode aumentar a suspeita. Nesses casos, a cintilografia pode ser um instrumento valioso e se mostrou altamente sensível ${ }^{(18)}$ para auxílio diagnóstico e localização da lesão. 
Leucemia - Pode também ser responsável por claudicação dolorosa em crianças $^{(19)}$, ainda nos seus primeiros passos. Além disso, o seu pico de incidência ocorre entre os dois e os cinco anos de idade, estando as queixas musculoesqueléticas presentes em cerca de $20 \%$ dos $\operatorname{casos}^{(20)}$.

O quadro clínico, pelo acometimento articular, dor óssea, febre e letargia, é semelhante ao da artrite e da osteomielite, estando seu diagnóstico diferencial associado à presença de outras alterações sistêmicas, como hepatoesplenomegalia, sufusões hemorrágicas $e$ sangramento ${ }^{(6)}$.

As radiografias, assim como no osteoma osteoide, podem ser normais, a cintilografia pode não constatar alterações e os exames laboratoriais na fase inicial também podem causar dúvidas, com elevação inespecífica do VHS e da contagem periférica de leucócitos ${ }^{(6,19,20)}$. Se os outros diagnósticos forem descartados e a suspeita persistir, a criança deve ser encaminhada a um hematologista para avaliação da medula óssea.

\section{PERÍODO ESCOLAR - QUATRO AOS 10 ANOS DE IDADE}

Nessa faixa etária as crianças tendem a ser mais cooperadoras durante o exame e já apresentam padrões mais maduros de marcha, facilitando a identificação das desordens. Além disso, as queixas são consideradas importantes nessa fase em que a criança está mais interessada em brincar e não tem desejo de se afastar de suas atividades habituais.

Em virtude da atividade vigorosa durante o dia e da fadiga muscular que a segue, são comuns queixas de dores noturnas e ao repouso, que genericamente são chamadas de "dor do crescimento"; no entanto, outras causas devem ser descartadas antes de se chegar a essa conclusão. Seu diagnóstico é eminentemente clínico ${ }^{(21)}$ e podem ser utilizados três critérios para ajudar a esclarecer se estamos diante de um caso de "dor do crescimento": a dor nas pernas é bilateral; ocorre somente à noite e não há queixas ou claudicação durante o dia, melhorando comumente com massagens e sem necessidade de medicação ${ }^{(7)}$.

As mesmas doenças já descritas anteriormente para crianças no início da idade da marcha devem ser lembradas, principalmente a sinovite transitória, que é mais frequente na idade entre três e oito anos e que é a causa de claudicação dolorosa mais comum durante toda a infância. Além dessas condições, três outras podem ter seu diagnóstico durante esse período: a doença de LeggCalvé-Perthes ${ }^{(22,23)}$, o menisco discoide e as discrepâncias de comprimento dos membros inferiores.
Doença de Legg-Calvé-Perthes - A doença de Perthes é caracterizada como a necrose avascular da cabeça do fêmur, sendo afecção de causa desconhecida e comportamento autolimitado, que acomete crianças entre os quatro e oito anos de idade ( $80 \%$ dos casos). É mais comum em meninos na proporção de 4:1. Em geral, a queixa é a claudicação, a dor não é frequente, mas quando presente, se manifesta na virilha, na coxa ou irradia-se para a face medial do joelho.

Ao exame pode ou não haver encurtamento, mas o principal sinal é a limitação da rotação interna do quadril.

Radiografias da bacia nas projeções em AP e Lauenstein ("posição de rã") devem ser solicitadas e o aspecto radiográfico nas fases iniciais, principalmente na projeção de "rã", aparece como uma linha translúcida subcondral (Figura 5), ou nas fases mais tardias podem ser observados o colapso e a fragmentação da epífise femoral proximal do fêmur, com zonas mais densas entremeadas por zonas radiolúcidas (Figura 6).

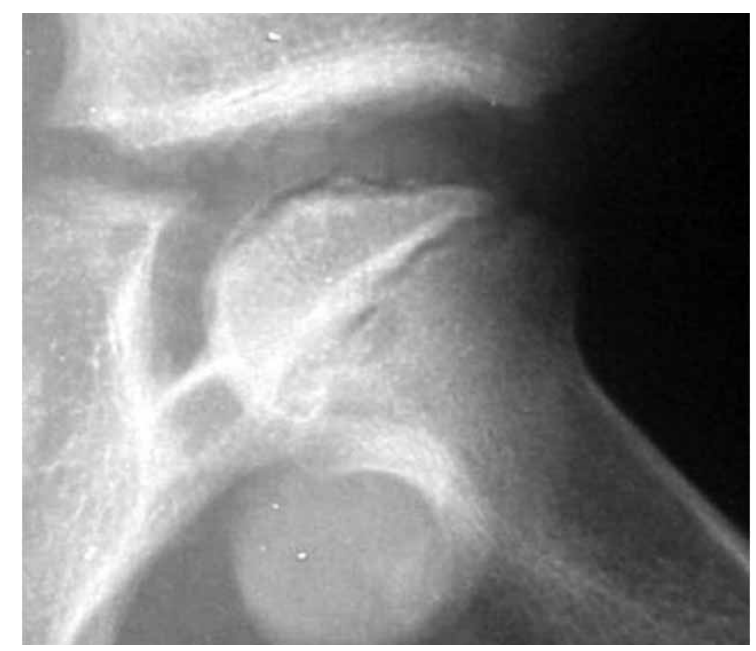

Figura 5 - Radiografia na "posição de rã", em que se evidencia a lise subcondral (sinal de Caffey) no quadril direito

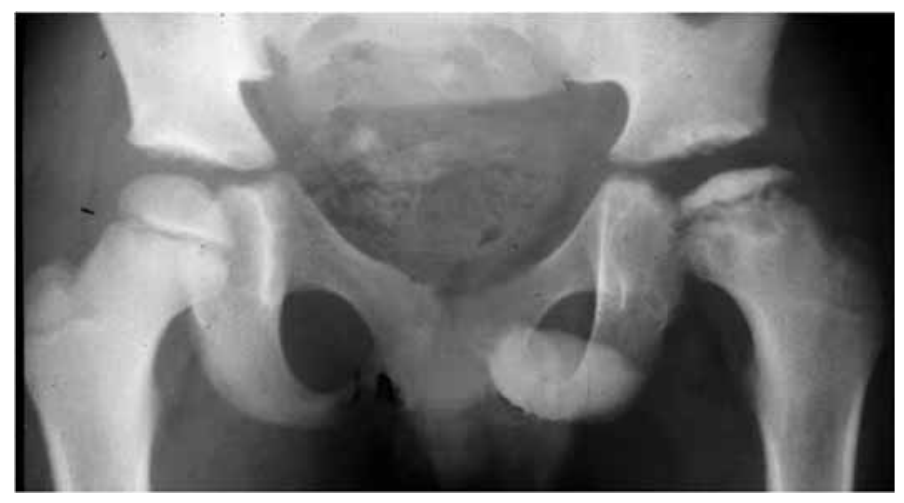

Figura 6 - Radiografia da pelve em AP, em que se observam o comprometimento do quadril esquerdo com diminuição da altura do núcleo ósseo e aumento da densidade com zonas de rarefação 
O tratamento para essa afecção é muito variável e a criança deve ser encaminhada ao ortopedista pediátrico para dar sequência ao tratamento, devendo ser orientada, de forma geral, à interrupção da descarga de peso no membro afetado, com a ajuda de muletas ou cadeira de rodas.

Menisco discoide - É uma deformidade congênita do menisco, que se encontra alargado e espessado, cobrindo total ou amplamente o planalto tibial lateral ou medial, sendo muito mais comum o primeiro ${ }^{(24)}$.

O menisco discoide costuma ser uma causa rara de claudicação dolorosa que piora com a atividade; além disso, o paciente costuma apresentar déficit de extensão total e a sensação de clique no joelho ${ }^{(25)}$.

A faixa etária em que a queixa clínica começa a se apresentar vai dos três aos 12 anos de idade, porém, é mais comum entre os oito e 12 anos $^{(26,27)}$. No exame físico há dor à palpação da interlinha articular lateral à pesquisa meniscal. As radiografias podem apresentar sinal indireto do problema pelo alargamento do espaço articular lateral, acompanhado do achatamento do côndilo femoral, mas causa dúvidas até para os médicos mais experientes, enquanto que a ressonância nuclear magnética (RNM) confirmará o diagnóstico, nos casos de forte suspeita(27).

Discrepância dos membros inferiores - A discrepância de comprimento dos membros inferiores se instala de forma progressiva e lenta, mostrando-se evidente nessa faixa de idade. Quando o acometimento é de um único membro, observa-se o apoio na ponta do pé na extremidade encurtada, realizado com o intuito de nivelar a pelve e manter o padrão de equilíbrio para a marcha.

A medida, com uma fita métrica, da distância entre a espinha ilíaca ântero-superior e o maléolo medial com os membros em extensão verifica se há ou não a diferença aparente de comprimento entre os membros inferiores.

Via de regra, a partir de $2,0 \mathrm{~cm}$, é considerada uma diferença clinicamente significativa e que precisa ao menos de acompanhamento ambulatorial. Para descobrir as causas da discrepância, uma radiografia panorâmica em ortostase dos membros inferiores (Figura 7) deve ser solicitada e esta pode fornecer informações tanto das prováveis causas (hemimelia fibular e fêmur curto congênito nas suas formas mais leves, fechamento precoce da placa de crescimento por trauma de impacção ou infecção frustra), quanto do segmento do membro que está acometido (fêmur proximal/distal ou tíbia proximal/distal).

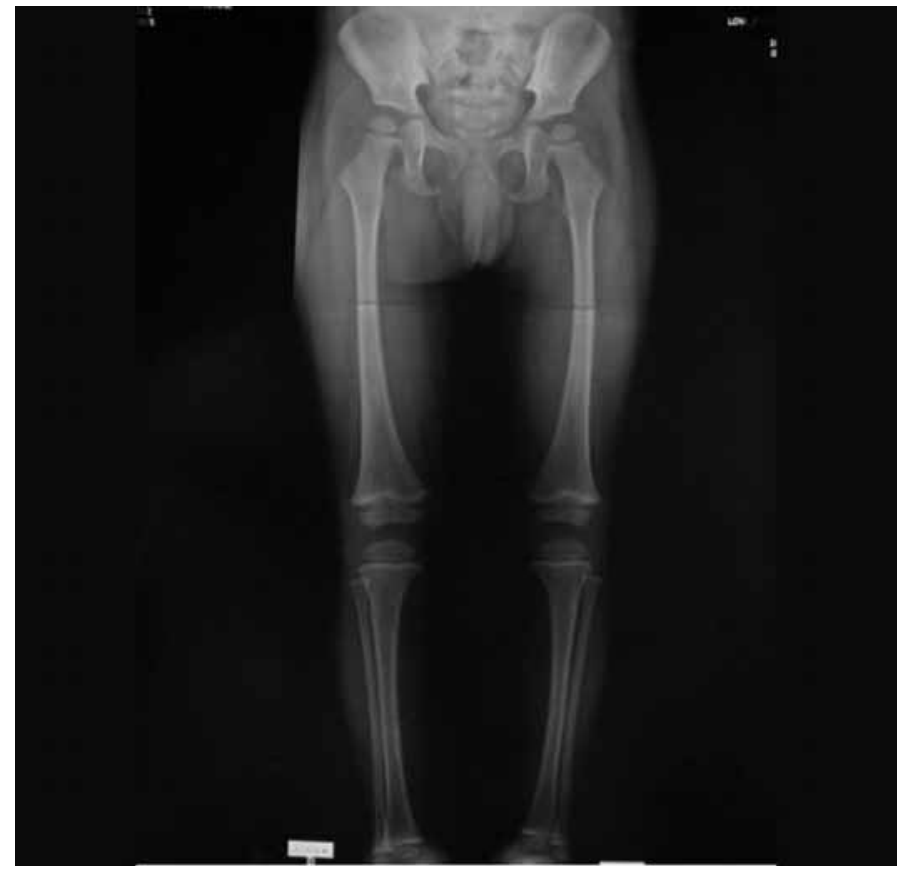

Figura 7 - Radiografia panorâmica dos MMII em ortostase

\section{PERÍODO ADOLESCENTE - DE 11 AOS 15 ANOS DE IDADE}

Pacientes nessa faixa etária comportam-se de forma diferente, com informações mais precisas, podendo ser colhida uma anamnese direta, com detalhes da sintomatologia, além de colaborarem para um exame físico mais completo.

Um cuidado que deve haver ao avaliar esses pacientes é ter em mente sua excessiva e compulsiva vontade de retornar à atividade esportiva, quando, então, tendem a minimizar o problema; ou, de outra forma, afastarem-se da atividade física, quando então maximizam o problema.

Diagnósticos mais prováveis nessa faixa incluem: escorregamento epifisário femoral proximal (epifisiólise), DDQ, condrólise, síndromes de overuse, osteocondrite dissecante e coalizão tarsal.

\section{DISTÚRBIOS DO QUADRIL}

Escorregamento epifisário femoral proximal (EEFP) - É a doença que mais comumente acomete o quadril do adolescente; ocorre na fase do estirão do crescimento e dois biótipos estão mais propensos, os baixos com sobrepeso e os altos e magros.

Acredita-se que exista íntima relação com a alteração hormonal que ocorre nessa faixa etária, pois pode estar associado ao hipotireoidismo, hipogonadismo e ao uso de suplementação com hormônio do crescimento $(G H)^{(28)}$. 
Pode ser bilateral em até $60 \%$ dos casos e é mais prevalente em meninos do que em meninas. A duração dos sintomas é geralmente de muitos meses e a queixa é a de dor leve, mas constante, na virilha, coxa ou joelho, que leva à claudicação dolorosa. Ao exame, a abdução e a rotação interna estão limitadas e quando se tenta fletir a extremidade inferior, esta frequentemente assume uma posição de rotação externa para permitir a progressão da flexão do quadril (sinal de Drehman). A dor pode se apresentar de forma aguda e intensa nas formas instáveis de escorregamento e, nesses casos, o prognóstico é pior. Radiografias em AP/Lauenstein da bacia definem o diagnóstico, observando-se translação e mudança na relação do colo com a cabeça femoral (Figuras 8 e 9). O tratamento é eminentemente cirúrgico.

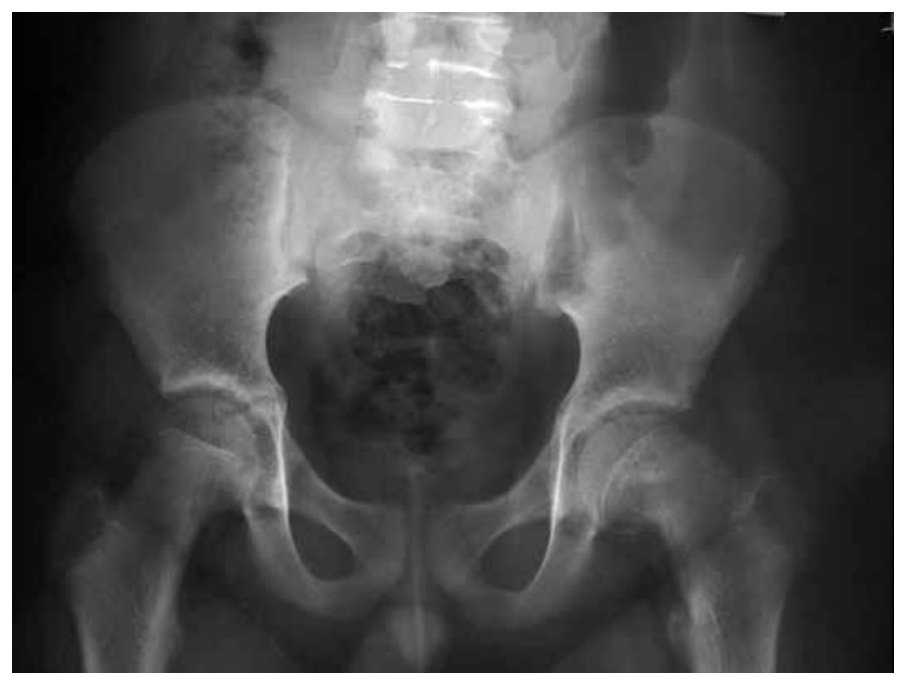

Figura 8 - Radiografia em AP onde se observa assimetria da altura epifisária

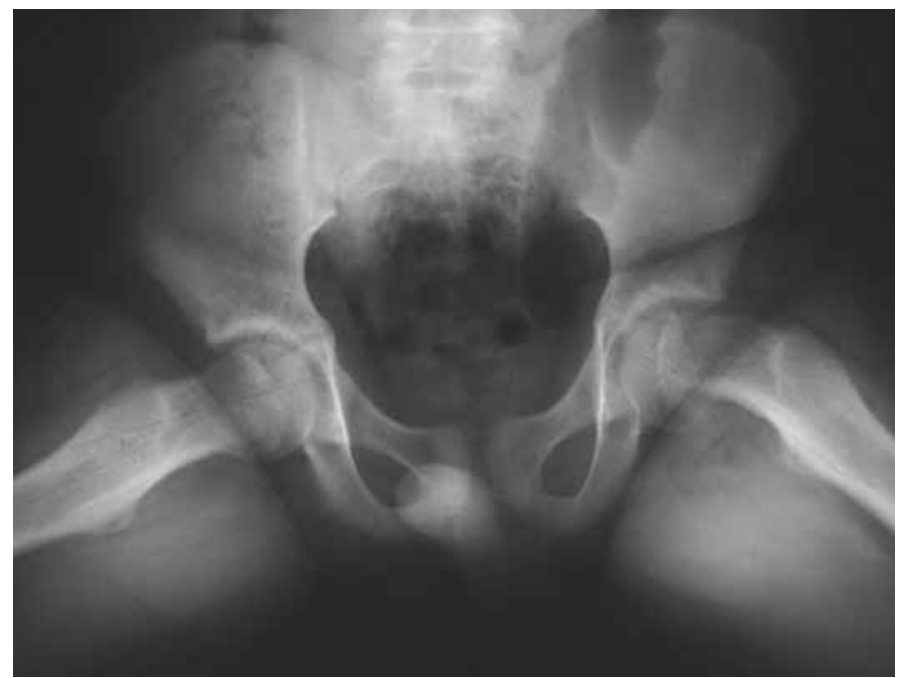

Figura 9 - Radiografia na "posição de rã" evidenciando o escorregamento do lado esquerdo

\section{Displasia do desenvolvimento do quadril (DDQ)} Pode ocorrer de a displasia de desenvolvimento do quadril tornar-se clinicamente sintomática durante a adolescência, sobretudo nos casos de subluxação. A criança pode ter estado aparentemente livre de qualquer desordem até essa fase e apresentar somente desconforto doloroso após atividade prolongada, que aumenta na adolescência e, assim, passa a apresentar claudicação dolorosa. O exame físico pode revelar pouco ou nada de anormal, sendo o diagnóstico novamente efetivado através de radiografias da bacia em AP e na "posição de rã" (Figura 10).

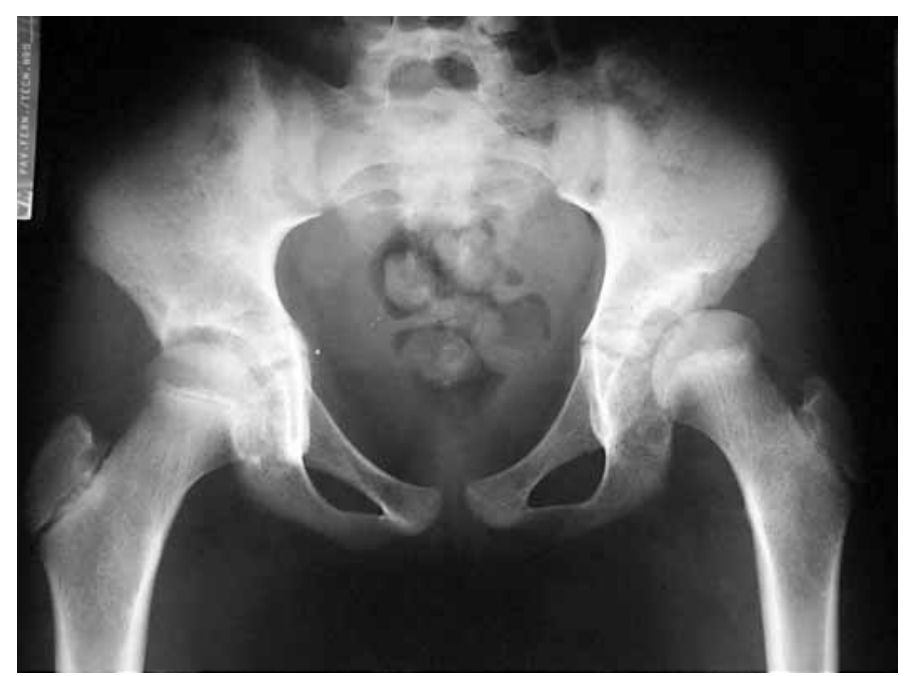

Figura 10 - Subluxação do quadril esquerdo de paciente adolescente, portador de DDQ

Condrólise - É a necrose da cartilagem articular do quadril; embora não seja uma doença comum, sua ocorrência está relacionada mais frequentemente com o EEFP e com o trauma; porém, há casos, não tão raros, em que a causa é desconhecida. Sabe-se que as meninas são acometidas cinco vezes mais que os meninos e que a idade de aparecimento está entre os 12 e os 14 anos. O quadro clínico é semelhante ao de outras afecções que acometem o quadril com dor na virilha, na coxa e/ou face medial do joelho, além de claudicação dolorosa, que pode variar de leve a intensa, porém, aqui a amplitude de movimentos está limitada em todas as direções. A radiografia da bacia em AP e na "posição de rã" é necessária para a confirmação diagnóstica com imagem; diminuição do espaço articular do quadril (mais de $2 \mathrm{~mm}$ de diferença entre um lado e outro), mais osteopenia de desuso e translucidez subcondral são os achados radiográficos.

O tratamento visa à melhora da sinovite irritativa, já que o processo de destruição articular é irreversível. 


\section{DISTÚRBIOS DO JOELHO}

Síndrome de "overuse" - Embora teoricamente qualquer parte do corpo que seja solicitada em excesso durante os exercícios possa entrar nesse conceito, o joelho é o sítio mais comum de acometimento. Essa é uma condição que tem sua incidência aumentada proporcionalmente ao aumento da atividade esportiva nessa faixa etária. Embora a dor seja o sintoma mais prevalente, a claudicação também se mostra como sinal comum. Fraturas por estresse da porção proximal da tíbia e fíbula, tendinite da patela e apofisite da tuberosidade anterior da tíbia (Osgood-Schlatter) são exemplos. História de atividade esportiva que piora a dor, melhorando com o repouso, e dor à palpação durante o exame levantam as suspeitas. Radiografias podem ser de difícil interpretação; na suspeita de fratura por estresse, a cintilografia pode ser útil. Na fase aguda, repouso, gelo e anti-inflamatórios constituem um bom tratamento inicial.

Osteocondrite dissecante - É uma condição que afeta a superfície articular com a separação de um fragmento localizado de cartilagem com osso subcondral adjacente. É a doença osteoarticular mais comum nos adolescentes e a dor é o sintoma típico, podendo ou não ser acompanhada de claudicação. Também está frequentemente relacionada com os esportes competitivos. O quadril e o tornozelo também podem ser afetados, mas o joelho é o sítio principal de acometimento, mais precisamente a porção lateral do côndilo femoral medial. O exame físico é inespecífico, sendo o diagnóstico realizado na maioria das vezes por radiografias (Figuras 11 e 12), que devem ser solicitadas nas incidências em AP/perfil e "túnel".

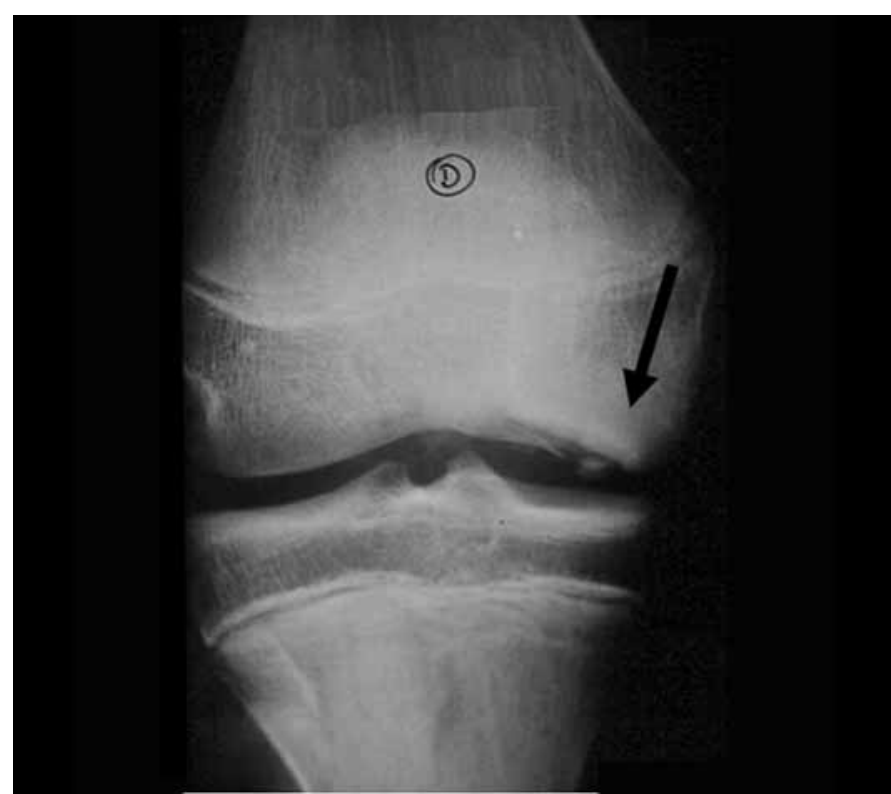

Figura 11 - Fragmento subcondral destacado

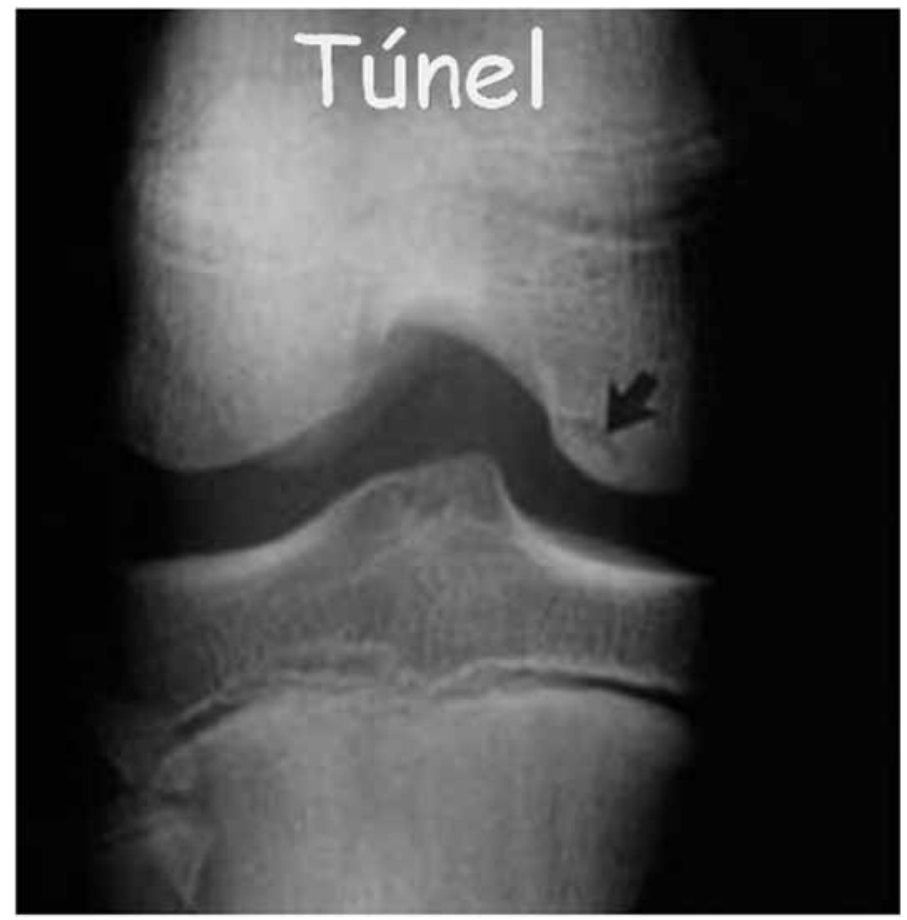

Figura 12 - Incidência radiográfica do túnel, que evidencia a lesão na porção lateral do côndilo femoral medial

Via de regra, o tratamento conservador resolve o problema, mas envolve, muitas vezes, mudança radical na vida do paciente, com restrição da atividade física por período relativamente prolongado (seis a oito semanas).

\section{OUTROS DISTÚRBIOS}

Coalizão tarsal - É caracterizada por fusão anormal entre dois ou mais ossos do tarso. Pode manifestar-se de forma precoce, nos primeiros anos de vida, mas o mais comum é tornar-se evidente clinicamente entre os 11 e 15 anos de idade, quando a coalizão inicialmente cartilaginosa começa a se calcificar, produzindo dor no seio do tarso, no dorso do pé ou no arco longitudinal e diminuição da mobilidade do pé, com claudicação. Pode ser bilateral em até $60 \%$ dos casos, manifestando-se em um único pé, principalmente durante a atividade física. Os músculos fibulares frequentemente encontram-se contraturados e espásticos, levando a pé plano, rígido e evertido. Quando o paciente é observado de costas e lhe é solicitado que fique apoiado sobre as pontas dos dedos, o pé falha "em varizar", o que indica rigidez da articulação subtalar. As coalizões mais frequentes são a talocalcaneana e a calcaneonavicular, podendo ser ósseas, fibrosas ou cartilagíneas. O diagnóstico, dependendo da localização e do tipo, pode ser feito mediante radiografias (Figura 13), ressonância magnética ou tomografia computadorizada (Figura 14). O tratamento é a ressecção da barra óssea. 


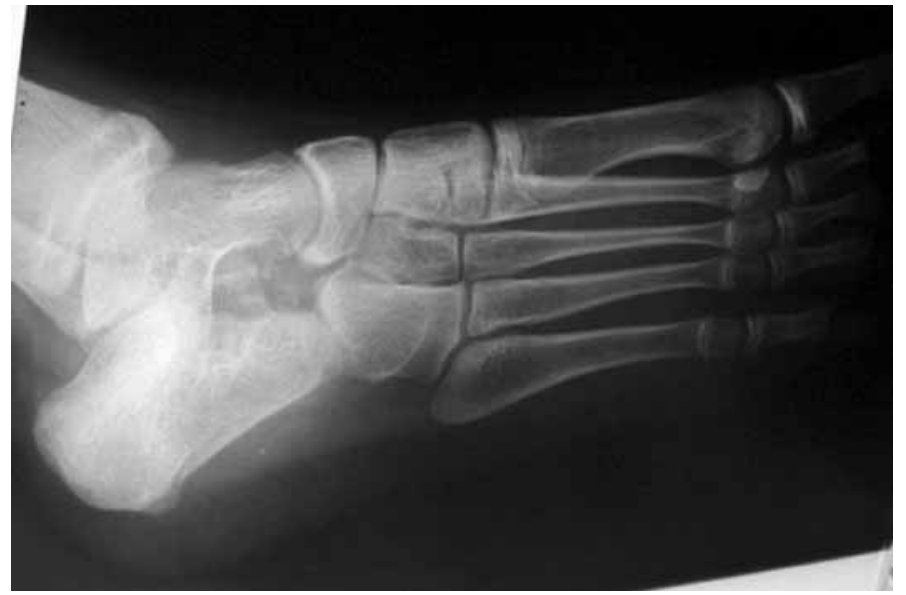

Figura 13 - Barra óssea talocalcaneana, vista na incidência radiográfica oblíqua do pé

\section{CONSIDERAÇÕES FINAIS}

A claudicação na criança é uma queixa comum e de difícil diagnóstico. É necessário ter em mente as principais doenças que causam este distúrbio, de acordo com o grupo etário e por frequência de aparecimento, para que se busquem detalhes da história referida pelo paciente e seus cuidadores e que se realize o exame físico dirigido para as afecções em questão, de forma

\section{REFERÊNCIAS}

1. Gibbons P. The limping child. Trauma. 2005;7(4):184-94.

2. Abbassian A. The limping child: a clinical approach to diagnosis. Br J Hosp Med (Lond). 2007;68(5):246-50.

3. Leung AKC, Lemay JF. The limping child. J Pediatr Health Care. 2004;18(5):219-23.

4. Philips WA. The child with a limp. Orthop Clin North Am. 1987;18(4):489-501.

5. Sawyer JR, Kapoor M. The limping child: a systematic approach to diagnosis. Am Fam Physician. 2009;79(3):215-24.

6. Richards BS. Claudicação na criança. In: AAOS - Atualização em conhecimentos ortopédicos - Pediatria. São Paulo: Atheneu; 2002. p.3-10.

7. Leet Al, Skaggs DL. Evaluation of the acutely limping child. Am Fam Physician. 2000;61(4):1011-18.

8. Aronson J, Garvin K, Seibert J, Glasier C, Tursky EA. Efficiency of the bone scan for occult limping toddlers. J Pediatr Orthop. 1992;12(1):38-44.

9. Blatt SD, Rosenthal BM, Barnhart DC. Diagnostic utility of lower extremity radiographs of young children with gait disturbance. Pediatrics. 1991; 87(2):138-40.

10. Beck RJ, Andriacchi TP, Kuo KN, Fermier RW, Galante JO. Changes in the gait patterns of growing children. J Bone Joint Surg Am. 1981;63(9):1452-6.

11. Boeck H, Vorlat P. Limping in childhood. Acta Orthop Belg. 2003;69(4):301-10.

12. Choban S, Killian JT. Evaluation of acute gait abnormalities in preschool children. J Pediatr Orthop. 1990;10(1):74-8.

13. Luhmann SJ, Jones A, Schootman M, Gordon JE, Schoenecker PL, Luhmann JD. Differentiation between septic arthritis and transient synovitis of the hip in children with clinical prediction algorithms. J Bone Joint Surg Am. 2004;86(5):956-62.

14. Caird MS, Flynn JM, Leung YL, Millman JE, D'Italia JG, Dormans JP. Factors distinguishing septic arthritis from transient synovitis of the hip in children: a prospective study. J Bone Joint Surg Am. 2006;88(6):1251-7.

15. Flynn JM, Widmann RF. The limping child: evaluation and diagnosis. J Am Acad Orthop Surg. 2001;9(2):89-98.

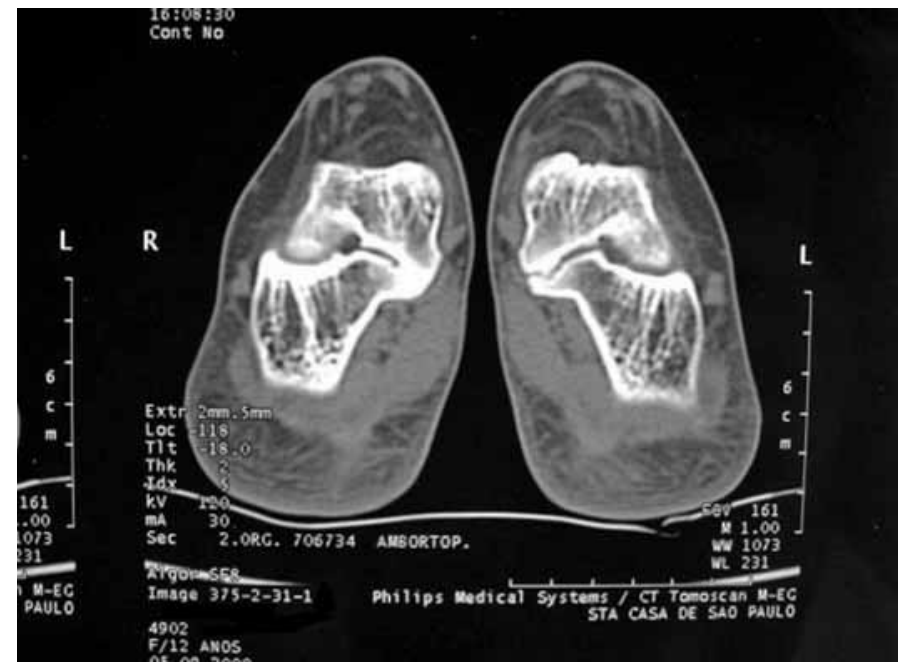

Figura 14 - Tomografia computadorizada mostrando barra óssea no pé $E$ e barra fibrosa no pé $D$

sistematizada. Solicitar exames adicionais conforme as principais suspeitas muitas vezes torna-se imprescindível para o correto diagnóstico. É importante que este seja realizado de forma precoce, pois o tratamento e o prognóstico das doenças envolvidas tomam rumos bem distintos e até desastrosos, caso haja atraso na definição terapêutica do caso.
16. MacEwen GD, Dehne R. The limping child. Pediatr Rev. 1991; 12(9):268-74.

17. Sussman M. Duchenne muscular dystrophy. J Am Acad Orthop Surg 2002;10:138-51.

18. Kaweblum M, Lehman WB, Bash J, Strongwater A, Grant AD. Osteoid osteoma under the age of five years: The difficulty of diagnosis. Clin Orthop Relat Res. 1993;(296):218-24

19. Tuten HR, Gabos PG, Kumar SJ, Harter GD. The limping child: a manifestation of acute leukemia. J Pediatr Orthop. 1998;18(5):625-9.

20. Rogalsky RJ, Black GB, Reed MH. Orthopaedic manifestations of leukemia in children. J Bone Joint Surg Am. 1986; 68(4):494-501

21. Asadi-Pooya AA, Bordbar MR. Are laboratory tests necessary in making the diagnosis of limb pains typical for growing pains in children? Pediatr Int. 2007; 49(6):833-5

22. Flynn JM, Widmann RF. The limping child: evaluation and diagnosis. J Am Acad Orthop Surg. 2001;9(2):89-98.

23. Fischer SU, Beattie TF. The limping child: epidemiology, assessment and outcome. J Bone Joint Surg Br. 1999;81(6):1029-34

24. Kelly BT, Green DW. Discoid lateral meniscus in children. Curr Opin Pediatr. 2002;14(1):54-61.

25. Kocher MS, Klingele K, Rassman SO. Meniscal disorders: normal, discoid and cysts. Orthop Clin North Am. 2003;34(3):329-40.

26. Aichroth PM, Patel DV, Marx CL. Congenital discoid lateral meniscus in children: a follow-up study and evolution of management. J Bone Joint Surg. 1991; 73(6):932-6.

27. Connolly B, Babyn PS, Wright JG, Thorner PS. Discoid meniscus in children: magnetic resonance imaging characteristics. Can Assoc Radiol J. 1996;47(5):347-54

28. Waisberg G, Braga SR. Epifisiolise. In: Cohen M. Tratado de ortopedia. São Paulo: Roca; 2007. p. 326-32. 